\title{
Transatlantica
}

Revue d'études américaines. American Studies Journal

\section{Thomas Bender, ed. Rethinking American History in a} Global Age.

Berkeley, CA : University of California Press, 2002. Ix + 427 pages. $\$ 22.50$.

\section{Marie-Jeanne Rossignol}

\section{(2) OpenEdition}

\section{Journals}

Édition électronique

URL : http://journals.openedition.org/transatlantica/677

DOI : $10.4000 /$ transatlantica. 677

ISSN : 1765-2766

Éditeur

AFEA

Référence électronique

Marie-Jeanne Rossignol, «Thomas Bender, ed. Rethinking American History in a Global Age. »,

Transatlantica [En ligne], 1 | 2003, mis en ligne le 05 avril 2006, consulté le 29 avril 2021. URL : http:// journals.openedition.org/transatlantica/677 ; DOI : https://doi.org/10.4000/transatlantica.677

Ce document a été généré automatiquement le 29 avril 2021.

\section{c) (i) $\Theta$}

Transatlantica - Revue d'études américaines est mis à disposition selon les termes de la licence Creative Commons Attribution - Pas d'Utilisation Commerciale - Pas de Modification 4.0 International. 


\title{
Thomas Bender, ed. Rethinking American History in a Global Age.
}

Berkeley, CA : University of California Press, 2002. Ix +427 pages. $\$ 22.50$.

\author{
Marie-Jeanne Rossignol
}

L'OAH, l'association américaine des historiens de l'Amérique, s'efforce depuis une vingtaine d'années d'internationaliser ses pratiques, sinon ses problématiques. «Internationaliser ", cela signifie, entre autres choses, s'ouvrir à des histoires et des auteurs non américains. La revue de l'association, le Journal of American History, est donc dotée d'un Advisory Board d'américanistes du monde entier ; sous l'impulsion d'un des anciens rédacteurs en chef du JAH, David Thelen, l'association décerne depuis le début des années 1990 un prix du meilleur livre étranger. David Thelen a également dirigé un numéro de la revue dédié à l'internationalisation de l'histoire américaine en décembre 1999. Il est donc tout naturel que l'OAH ait accueilli favorablement le «Project on Internationalizing the Study of American History » de Thomas Bender, de New York University. Autour d'une large question ( $«$ How does one frame the narrative of American history in the context of a self-conscious global age?») se sont donc réunis de 1997 à 2000, à La Pietra en Italie, lors de 4 séminaires, des participants américains et non-américains, spécialistes d'histoire des Etats-Unis comme d'autres régions du monde, pour repenser le récit national américain à une époque où la catégorie du «national», longtemps structurante et dominante en histoire, subit de nombreuses critiques et ne parait plus pouvoir rendre compte seule des expériences américaines, ou d'autres nationalités. L'ouvrage collectif qui a été tiré de ces rencontres constitue une somme très précieuse dont il est difficile de rendre ici pleinement compte tant elle est dense et riche. La lecture en est pourtant en général aisée car il s'agit d'un débat sur un sujet qui concerne l'avenir de la profession historique aux Etats-Unis ; les auteurs sont souvent passionnés, et leurs développements parfois polémiques.

Si d'aucuns en doutaient, l'internationalisation de l'histoire américaine a déjà largement été entamée et Rethinking American History in a Global Age ne fait que le rappeler en passant en revue les différentes évolutions historiographiques des vingt dernières années qui ont tout simplement rendu impossible l'écriture d'une histoire 
américaine insulaire, refermée sur un cadre étroitement national: l'histoire des relations internationales a pris en compte les forces et les acteurs transnationaux, ainsi que les identités non-nationales (Akira Iriye); les historiens de l'esclavage et des Africains-Américains, comme ceux de l'immigration ont quitté le cadre des Etats-Unis pour recentrer leur récit sur l'Atlantique et s'intéresser aux communautés dans leur dimension diasporique (Charles Bright et Michael Geyer, Robin G. Kelley); les spécialistes de l'histoire coloniale du continent nord-américain ont retracé les contacts multiples entre Amérindiens et colons de diverses nationalités qui se nouaient du Nord au Sud, d'Ouest en Est, faisant du cœur du continent un haut lieu de la diplomatie internationale et des échanges culturels (Karen Ordhal Kupperman). Toutes ces approches se sont appuyées sur d'autres notions que la nation; elles ont remis au goût du jour l'étude des frontières américaines dans l'histoire, mais dans une toute autre perspective que celle de Turner, pour montrer qu'elles étaient perméables, franchissables, modulables et modelables. En liant l'histoire des Etats-Unis à celle des Caraïbes et de l'Europe, ces nouvelles histoires américaines ont mis à bas l'idée d'une Amérique différente, meilleure, exceptionnelle enfin. Des comparaisons moins usitées, avec d'autres sociétés de colonisation, telles l'Australie, permettraient de remettre également en question le rapport des Américains à leur environnement, moins unique qu'ils ne le croient, et de replacer le développement économique de telles régions périphériques dans le cadre de l'économie-monde (Tyrell).

Quitter le cadre du récit national, ainsi que le font ces adeptes de problématiques hybrides, créolisées et croisées, permet donc aux historiens américains de critiquer et de dépasser la perspective nationaliste antérieure, mais Thomas Bender ne plaide pas pour une histoire "postnationale» des Etats-Unis, il milite simplement pour une histoire moins «provinciale» qui enrichirait le récit national en lui donnant plus d'épaisseur, et plus de résonance internationale. Il est suivi sur ce plan par plusieurs historiens qui proposent des thématiques «transnationales » pour l'étude de l'histoire nord-américaine : Dirk Hoerder trace les grandes lignes d'une approche comparée des migrations en Amérique du Nord, de l'Atlantique au Pacifique; Robert Wiebe suggère, parmi plusieurs cadres d'analyse, d'étudier «la démocratie, le nationalisme et le socialisme » comme « des aspects interactifs d'un processus transocéanique » du XVIIIè au XXè siècle; enfin, toujours dans le domaine de l'histoire intellectuelle transatlantique, Daniel T. Rodgers voudrait que l'on se penche davantage sur « the Age of Social Politics ", des années 1890 à 1940, où s'élaborèrent, dans un trafic constant d'idées et de perceptions, les nouvelles exigences des réformateurs sociaux, d'un côté à l'autre de l'Atlantique.

Face à ce déferlement de projets tous plus comparatistes et moins américano-centristes les uns que les autres (et donc extrêmement sympathiques, disons-le), quelques articles prennent le contre-pied du parti pris anti-exceptionaliste du projet pour s'inquiéter de cette attitude angéliste, qu'ils jugent déconnectée des enjeux du monde réel : Marilyn B. Young rappelle que l'idéologie américaine, telle qu'elle se reflète dans le discours public et les représentations majoritaires, reste profondément structurée par une vision exceptionaliste qui se double, depuis plus d'un siècle, d'une puissance incontestée. Il ne faudrait pas que de nouveaux récits de l'expérience nationale, sous prétexte de décentrement et de comparaison, en gomment les abus exceptionnels. A tous ses collègues, elle lance cette mise en garde qu'il faut citer: "Efforts to 
internationalize America's history, to diversify and multiply its culture, need to keep in mind the reality of American hegemony and its dominant, self-absorbed culture. ( ...). Decentering America in one's head is a good thing. But it does not in itself create a world free of its overwhelming military and economic power, and it is crucial to remember the difference or the effort to decenter American history will run the danger of obscuring what it means to illuminate. (291)» David Hollinger, dans une veine similaire, pense qu'on ne peut évacuer la nation du récit de l'histoire américaine, au risque de ne plus pouvoir évaluer le coût humain considérable de cette construction nationale (esclavage, extermination des Indiens, exclusion des Hispaniques). Redonner la parole aux exclus, est-ce vraiment leur donner a posteriori le pouvoir, est-ce là la mission de l'historien? Ou alors faut-il continuer à étudier les dirigeants de la nation pour mieux défendre les minorités opprimées?

A ces remises en cause des problématiques anti-exceptionalistes ou transnationales, s'ajoute une autre série d'articles critiques du processus d'internationalisation, qui porte, entre autres choses, sur l'authenticité de l'ouverture de la profession aux historiens américanistes non-américains (François Weil et Ron Robin). L'article de François Weil est exemplaire sur ce sujet, en ce qu'il utilise sa connaissance exhaustive de l'historiographie nord-américaine pour aboutir à des conclusions que les autres historiens français (mais aussi européens) de l'Amérique subodorent depuis longtemps mais n'auraient pu si bien étayer : les américanistes étrangers sont bien des Extérieurs à un milieu américain dont la principale caractéristique est l'insularité. Insularité due à la structuration très professionnelle du milieu. Pour pénétrer le milieu américain, les américanistes non-américains doivent adopter ses problématiques, et son mode de travail, mais à quel prix, en termes d'originalité ? On peut se demander ce qu'ils pourraient alors apporter aux Américains: c'est un cercle vicieux. A l'inverse, l'historiographie américaine, produite en circuit fermé, n'a pas d'impact sur les historiographies d'autres pays, alors que les historiens américains de la France ont su s'y faire apprécier en s'ouvrant aux travaux français tout en apportant leur propre contribution.

L'internationalisation de l'histoire américaine, dans ses pratiques comme dans ses problématiques, est donc une affaire plus compliquée que les efforts initiaux de l'OAH n'auraient pu le laisser penser. Ce livre en reflète toute la complexité.

\section{INDEX}

Thèmes : Recensions 
AUTEUR

MARIE-JEANNE ROSSIGNOL

Université Paris 7 - Denis Diderot 